\title{
Correction to: State-Level Variation in Vocational Rehabilitation Service Use and Related Outcomes Among Transition-Age Youth on the Autism Spectrum
}

\author{
Anne M. Roux ${ }^{1}{ }^{10} \cdot$ Jessica E. Rast ${ }^{1} \cdot$ Paul T. Shattuck $^{1}$
}

Published online: 4 April 2019

(c) Springer Science+Business Media, LLC, part of Springer Nature 2019

\section{Correction to: \\ Journal of Autism and Developmental Disorders \\ https://doi.org/10.1007/s10803-018-3793-5}

The original version of this article unfortunately contained mistakes in Table 1 values. Some of the values in "TAYASD who received services" were incorrect. The corrected Table 1 is given below.

Publisher's Note Springer Nature remains neutral with regard to jurisdictional claims in published maps and institutional affiliations.

The original article can be found online at https://doi.org/10.1007/ s10803-018-3793-5.

Anne M. Roux

amr376@drexel.edu

Jessica E. Rast

jer336@drexel.edu

Paul T. Shattuck

pts33@drexel.edu

1 A.J. Drexel Autism Institute, Drexel University, 3020 Market St., Suite 560, Philadelphia, PA 19130, USA 
Table 1 Characteristics of transition-aged youth on the autism spectrum (TAY-ASD) who received services from Vocational Rehabilitation before case closure FFY 2014-2016

\begin{tabular}{|c|c|c|}
\hline & $\begin{array}{l}\text { TAY-ASD who received } \\
\text { services }(n=35,823)\end{array}$ & $\begin{array}{l}\text { TAY-ASD who } \\
\text { received services } \\
(\%)\end{array}$ \\
\hline Male & 29,906 & 83.5 \\
\hline \multicolumn{3}{|l|}{ Age at application } \\
\hline $14-15$ & 556 & 1.6 \\
\hline 16 & 2490 & 7.0 \\
\hline 17 & 6777 & 18.9 \\
\hline 18 & 8258 & 23.1 \\
\hline 19 & 4947 & 13.8 \\
\hline 20 & 3972 & 11.1 \\
\hline 21 & 3274 & 9.1 \\
\hline 22 & 2261 & 6.3 \\
\hline 23 & 1814 & 5.1 \\
\hline 24 & 1474 & 4.1 \\
\hline \multicolumn{3}{|l|}{ Race } \\
\hline White & 30,252 & 84.5 \\
\hline Black & 3605 & 10.1 \\
\hline Other/multiple & 1966 & 5.6 \\
\hline Ethnicity-Hispanic or Latino & 2541 & 7.1 \\
\hline \multicolumn{3}{|l|}{ Primary source of support at application } \\
\hline Personal income (earnings, interest, dividends, rent) & 1048 & 2.9 \\
\hline Family and Friends & 26,789 & 74.9 \\
\hline Public support (SSI, SSDI, TANF, etc.) & 7535 & 21.0 \\
\hline $\begin{array}{l}\text { All other sources (e.g., private disability insurance and } \\
\text { private charities) }\end{array}$ & 451 & 1.3 \\
\hline \multicolumn{3}{|l|}{ Type of health insurance at time of application } \\
\hline Private & 15,257 & 42.6 \\
\hline Public & 11,266 & 31.5 \\
\hline Both private and public & 2121 & 5.9 \\
\hline No insurance & 7179 & 20.0 \\
\hline
\end{tabular}

SSI supplemental security income, SSDI social security disability insurance, TANF temporary assistance for needy families 